\title{
Innovative Teaching Mode Applied in the Course of Digital Media Technology
}

\author{
F.Q. ZHANG, J.J. Guo, B. Wang, Y. Liu \& Z.Y. Yu \\ College of Computer Science, North China University of Technology, Beijing, China
}

\begin{abstract}
Digital Media Technologies is a thriving discipline in IT field, with its academic and applied value widely acknowledged. Digital Media Technologies course composed thereof, requires rich information in theory and application. With an aim to refine the innovative teaching methods in the course, this paper summarizes years of teaching experiences, and compares domestic researches carried out in colleges and universities, and analyzed key technologies in this field. The focus is located on how to inspire innovation awareness and practice in teaching activities. Last, it presents some ideas on how to further improve the quality of teaching.
\end{abstract}

KEYWORD: Digital Media Technologies; Teaching Practice; Innovation mode; Teaching Quality

\section{INTRODUCTION}

Mainly based on computer graphics and computer image processing, Digital Media Technologies is an emerging technology which takes computer collection, storage, processing and transmission of text, graphics, images, sound, video and animation as handling objects. Digital media, which feature in rich content, strong performance, ease of storage and transport, have become significant means in promoting economic development. Digital media technologies are playing an increasingly important role in digital design and quality inspection of automobile industries, shipbuilding, and equipment manufacturing. To meet people's ever-growing spiritual and cultural needs, digital media provide technical support for the development of cultural and creative industries, in particular digital animation industry. Imagination and creativity the digital animation production can further strengthened to enhance the content and its audio-visual effects.

With a focus on hot research area, this paper first introduces key technologies recently applied, compares the research progress of different universities, and then from the perspective of teaching, explores the innovation mode in digital media technologies class, focusing on the analysis of how to achieve mastery and inspire innovation through comprehensive study activities. Finally, some suggestions on how to further improve teaching quality are put forward.

\section{KEY TECHNIQUES IN DIGITAL MEDIA TECHNOLOGIES}

\subsection{The processing, retrieval and synthesis of media content}

The processing, retrieval and synthesis of media content are key techniques of Digital Media Technologies. One major application is constructing digital media library for television advertising design, animation design and so on. On one hand, we can retrieve media material from massive media data to serve as raw materials of design process. On the other, we can further enhance the fidelity of digital production during the production process, once we completed the design. The existing media content processing, analysis and retrieval methods differ greatly for different media carriers. For different media types, researchers have attempted to extract the visual and auditory features which can efficiently characterize the media content, and sometimes transform and enhance the characterization depending on different application requirements, and establish correspondence between features and high-level concepts underlying the use of machine learning methods.

\section{$2.23 D$ modeling}

Building realistic virtual environment with the help of computers is another key technique of Digital Media Technologies. It enables us to break the traditional time and space limitations and meet the 
special needs of creative design. And most importantly, it directly determines the degree of realism of creative works based on digital media technologies. Currently, facing with the demand of larger, more detailed and more realistic special effect in the application of three-dimensional scenes, researchers have already done a remarkable work. As for efficient modeling, the rapid modeling based on measurements is widely used. The current study primarily focus on automatic splicing of massive point cloud data, semantic-based model repairing and maintaining the geometry of the details of the deformation, realistic material modeling, modeling and simulation of fluid and flexible objects etc.

\subsection{Generation and interactive of $V R$}

The actual situation fusion technology is an important approach to enhance the fidelity of digital media content and user immersion. It is committed to combine a computer-generated virtual scene with real objective existence in the same digital media space, presenting a real world with virtual objects. The interactive strategy based on the combination of virtual and reality has become a hot research topic at home and abroad. And the recent progress and developments are mainly gathered in the following five directions, virtual $3 \mathrm{D}$ registration and space occlusion handling, color fusion and unification of virtual graphics objects and real video images, scene virtual/real light fusion and shadow processing based on the target light, image \& video material processing of the combined scene, interface design and interaction mechanism study on the combined scene, users' perception analysis and reliability evaluation.

\section{RESEARCH PROGRESS OF DIGITAL MEDIA IN CHINESE UNIVERSITY}

Tsinghua University Visual Media Research Center carried out theory and methodology research of mass visual media intelligent processing under the network environment. The team has begun to make important progress in efficient visual perception computation and analytical learning in mass network information, adapted to the available sense of human visual media interaction, fusion and presence, association mining of multi-source visual media etc. Published papers thereof are on internet-based analysis, processing and retrieval for image's content, composing a new picture from a simple freehand sketch annotated with text labels, semiautomatic image editing framework dedicated to individual structured object replacement from groups[Tao 2009, Tao 2013, Zhang 2012].

Beijing University of Aeronautics and Astronautics Beijing Key Laboratory of Digital
Media has performed research on analysis, processing and retrieval of media's content, delved into cognitive mechanism of digital media and the new theory, methods of analyzing and processing of digital media, put forward a cognition model based on hierarchy and integration combination, and perfected hierarchical representation and description framework of media objects. Beijing University State Key Laboratory of Virtual Reality Technology and System developed the ancient Chinese architecture modeling technique based on drawing of ancient Chinese architecture. Semantic analysis is applied to extract construction rules to recognize individual construction components, and to build an edible 3D model which can be used to for rapid generation of ancient architectures [Fei 2012, Yang 2012].

In the aspect of architecture and urban environment modeling based on drawing/image, Hong Kong University of Science and Technology used continuous image sequence captured to generate street-side 3D models. [Jian 2009] Zhejiang University CAD/CG State Key Laboratory conducted research on processing of geometrical data and skin deformation. They present a cagebased method for transferring animation from a mesh sequence or motion capture data to geometric models in variant representations. The animation data could be reused. Besides, they also present an geometric deformation method to represent animation of water drops, more precise in displaying the process of water drop deformed by tension force[Lu 2010, Yi 2012].

Nevertheless, when it comes to the combination of research technology and real industry, there are still problems remains to be solved, especially digital media technique and cultural creation industry. At present, Domestic researchers are more focused on national great strategic demand and tackling key fields of technology. Scarce progress has been made in cultural creative industry. Chinese research institutions such as Tsinghua University, Beijing University of Aeronautics and Astronautics, Zhejiang University, Chinese Academy of Sciences have carried out fruitful researches, and published many excellent papers and articles at famous international conferences or periodicals. However, their achievements are rarely used in cultural creative industry such as film and cartoon production. In sharp contrast, latest research results published in academic circles by American universities or research institution are often seen in American movies. For example, Light Stage System, one project of University of Southern California Institute for Creative Technologies, was used on several famous American films such as Spider-Man 2 (2004), Superman Returns (2006), Avatar (2009), The Curious Case of Benjamin Button (2008), bringing back Oscar for visual effects[Paul 2012]. 


\section{COURSE FEATURE OF DIGITAL MEDIA TECHNOLOGIES}

The summary of the latest developments of digital media technologies shows a comprehensive and overlapping nature of the discipline. It focuses on the integration of computer application, cybernetics, graphic images, database design, parallel computing, virtual reality technology, and media arts. Theories, methods and techniques of digital media technologies courses cover mathematical concepts and physical laws. Numerical calculation, differential equations, and the formula of mechanics are all prerequisite for 3D modeling. Students are required to have a solid knowledge base in math and physics. Digital media technologies can simulate nonexistent substance in the real world, and interactive experiences can be drawn with the generated virtual environment. Design, training, and evaluation under the virtual environment can inspire students with innovation thoughts. The course covers various theories and many typical algorithms and uses the development environment and tools. Software and hardware combination accounts for a large proportion of the teaching process.

\section{TEACHING INNOVATION PATTERN}

\subsection{Emphasis on basics}

For learning, clear explanation of the basic concepts, methods and algorithms comes first. Digital Media Technologies course consist of different disciplines and fields and these theories may have little relevance. For complex theories, we need to trace back to itself with emphasis on the basics. In teaching, a knowledge pyramid can be built with layers and difficulties. Due to time limits, in-depth teaching is not realistic for college class. Emphasis need to be located on the basic and the domestic and international hot issues. On the tool selection, we should give priority to the mainstream open source tools and combine them with projects. Priorities can be given to the concept present in various topics to deepen students' understanding of the concept and then move to the complex theory. Such teaching methods can be applied throughout the teaching process to achieve mastery.

\subsection{Interactive teaching}

Interactive teaching is an effective teaching pattern for digital media technologies courses. It is studentoriented, which can exercise the students to analyze and solve problems effectively. For example, according to the students' interests and characteristics, we can use group discussion. By debating and expressing their views, students are inspired with enthusiasm. In this pattern, teachers act as the organizers of interaction. They can constantly present questions to promote further discussion. Teachers may have targeted selection of students to speak to control the discuss progress by observing students' understanding of knowledge. Interactive teaching requires teachers to summarize the issues discussed, answer questions, and help to achieve a better understanding.

\subsection{Case driven, cognition and practice}

Case test is an effective way to examine students' mastery of knowledge. In digital media technologies courses, specific knowledge topics can be combined into the case. Cases can be categorized into hardware, categories, modeling, rendering, animation, interactivity, etc, covering key elements of digital media techniques. Through these cases, theoretical knowledge acquired can be applied to concrete practice to deepen understanding. In addition, according to characteristics of research and engineering, cases can be divided into algorithm type and program type. Students are encouraged to read latest literature, improve the algorithm and stimulate academic innovation. Digital Media Technologies involves many disciplines which means program implementation requires a solid grasp of programming ability. The selection of appropriate system development platform, hardware description language, scripting languages, open source engine should be case-oriented. Cases developing process can practice students' problem solving ability, and at the same time examine their understanding of the theoretical knowledge.

\section{CONCLUSION}

The course of "Digital Media Technologies" features in comprehensive theories, creative thinking, and rich engineering applications. Its teaching process requires updated methods. First, teaching objectives should be defined based on the basic theories and students can be categorized according to their specific interests. Then by using interactive teaching pattern, we give priority to students and apply their knowledge to practice. Finally, we use cases to exercise students' practice ability. For undergraduates, focus should be located on their interest for the purpose of building a solid theoretical foundation. For graduate students, academic research and engineering applications should be introduced for guidance to lay a solid foundation for next level of research and knowledge.

\section{ACKNOWLEDGEMENTS}

This research work is supported by Foundation for 
Social Science of Ministry of Education (NO.14YJCZH200).

\section{REFERENCES}

[1] Tao C, Ming M. C, Ping T. 2009. Ariel Shamir, Shi-Min $\mathrm{Hu}$, Sketch-Photo: Internet Image Montage, ACM Transactions on Graphics, 28(5), Article No. 124.

[2] Tao C, Ping T, Li Q. M. Ming M C. 2013. A Human Image Database and Personalized Content Synthesis, IEEE Transactions on Visualization and Computer Graphics, 19(5):824-837.

[3] Zhang F.L, Ming M C, Shi M.H. 2012. ImageAdmixture: Putting Together Dissimilar Objects from Groups, IEEE Transactions on Visualization and Computer Graphics, 18(11):1849-1857.
[4] Fei H, Yue Q, Hong Q. 2012. Drawing-Based Procedural Modeling of Chinese Architectures. IEEE Transactions on Visualization and Computer Graphics. 18(1):30-42.

[5] YANG S, QI Y, QIN H. 2012. Simultaneous Structure and Geometry Detail Completion Based on Interactive User Sketches. Science in China Series F: Information Sciences, 55(5):1123-1137.

[6] Jian X.X, Tian F, Peng Z, Maxime L and Long Q. 2009. Image-based street-side city modeling. ACM Trans. Graph. 28(5).

[7] Lu C, Jin H, Han Q S, and Hu J B. 2010. Technical Section: Cage-based deformation transfer. Comput. Graph. 34(2): 107-118.

[8] Yi Z Z, H M. W, Shuai W, Yi Y.T, and Kun Z. 2012. A Deformable Surface Model for Real-Time Water Drop Animation. IEEE Transactions on Visualization and Computer Graphics 18(8):1281-1289.

[9] Paul D. 2012. The Light Stages and Their Applications to Photoreal Digital Actors. SIGGRAPH Asia. 